\title{
Editorial
}

\section{¿Qué nos falta por hacer en la Prevención Cardiovascular?}

Tomás Romero

\author{
Clinical Professor, School of Medicine, Department of Cardiology, \\ University of California, San Diego. USA.
}

El trabajo de Bambs y colaboradores, "Facilitators and barriers to the adoption of healthy lifestyles after first myocardial infarction in Chile: A qualitative study", recientemente publicado en esta revista ${ }^{1}$, plantea interrogantes que aun buscan respuestas en la prevención cardiovascular secundaria: ¿cuáles son los métodos más eficaces para implementar o resolver los factores que facilitan o dificultan la adopción de estilos de vida favorables después de un evento coronario agudo? Estos cambios están destinados a contribuir en el control de algunos de los factores mayores de riesgo cardiovascular (tabaquismo, hipertensión, obesidad, inactividad física).
Un estudio que analizó los factores incluidos en la reducción de la mortalidad por enfermedad coronaria en los EEUU entre los años 1980 y 2000 atribuyó un $44 \%$ de esa reducción al control de los factores mayores de riesgo cardiovascular ${ }^{2}$. Como lo señalan los autores, estudios en Chile, Estados Unidos y Europa que han evaluado la respuesta a las recomendaciones de cambios a conductas y hábitos desfavorables en los pacientes después de un infarto del miocardio han demostrado hasta ahora un éxito limitado (no mayor del 50\%) e ilustrado la necesidad de mayor información acerca de los factores facilitadores o obstaculizadores de estos cambios ${ }^{3-4}$.

\section{Correspondencia:}

Tomás Romero, MD.

Clinical Professor, School of Medicine, Department of Cardiology,

University of California, San Diego.

Institutional Review Board, Sharp Health Care,

San Diego, California

tomas.romero@sharp.com 
Bambs y colaboradores crearon una red informativa utilizando entrevistas a los actores directos del evento coronario (21 pacientes sufriendo un primer Infarto agudo de Miocardio) más 6 cardiólogos practicando en la misma red de servicios hospitalarios en que fueron atendidos los pacientes y 8 expertos nacionales en cardiología preventiva, todos ellos no directamente participando en el manejo de esos pacientes. Esta información fue organizada y analizada de acuerdo a la metodología cualitativa propuesta en la Teoría Fundamentada (Grounded Theory), a través de la cual se generan en forma inductiva categorías de datos obtenidos empíricamente mediante cuestionarios/entrevistas de los participantes e intentar, de esta manera, la integración y uso coherente de esa información ${ }^{5}$. Un importante aspecto de la metodología usada fue la selección de pacientes que representaran una máxima diversidad demográfica (edad, sexo), socioeconómica (educación) y clínica (modalidad de revascularización) y limitar el número de sujetos reclutados cuando se llega al punto de saturación informativa de los ya entrevistados. El estudio destaca una serie de factores individuales, familiares, laborales, comunitarios/ambientales, relacionados con los proveedores de salud que pueden actuar facilitando o dificultando la adopción de estilos de vida favorables. Algunos han sido ya mencionados anteriormente por otros: entre los factores facilitadores, una actitud optimista, alto nivel de educación, apoyo familiar, retorno al trabajo, buena relación médico-paciente.

Aspectos menos conocidos que aporta este estudio son el apoyo emocional que perciben los pacientes al participar en actividades de grupo y en lograr la adopción de conductas favorables (por ejemplo, dejar de fumar, empezar a caminar diariamente, bajar de peso), lo que ha sido destacado en las primeras publicaciones de Chile y EEUU que describieron a la rehabilitación cardíaca como herramienta importante en la prevención secundaria después de un infarto del miocardio $^{6-7}$. Algunos pacientes (especialmente jóvenes) mencio- naron un aspecto negativo del retorno al trabajo, importante de considerar: las responsabilidades económicas en el grupo familiar y la carga laboral y stress emocional consiguientes pueden constituir una barrera en la adopción de conductas y hábitos favorables, como, por ejemplo, poder dedicar tiempo suficiente a una actividad física regular.

Otro aspecto importante de este trabajo fue la identificación de una actitud escéptica por parte de los médicos acerca de la efectividad de lograr cambios en estilos de vida desfavorables en sus pacientes, percepción en parte apoyada por sus propias experiencias personales. Esta percepción de los médicos contrasta con la sobrevaloración que ellos tienen de la efectividad de los tratamientos farmacológicos e intervencionales en el manejo de la enfermedad coronaria, en parte producto de una formación como especialistas a menudo sesgada hacia lo tecnológico en desmedro de los aspectos preventivos y en los métodos de interacción y comunicación eficaz con los pacientes.

Un aporte mayor de este trabajo fue identificar falencias importantes en el apoyo de pacientes después de un infarto del miocardio. Una de ellas es la falta de acceso garantizado a programas de rehabilitación cardíaca (ninguno de los pacientes incluidos en este estudio la recibió), problema que no es exclusivo de Chile sino que afecta incluso a países de alto desarrollo económico ${ }^{8}$.

El tema de fondo en la adopción de conductas favorables después de un infarto del miocardio es la educación y motivación del paciente, tarea que es responsabilidad primaria del médico y otros proveedores de salud asociados en su manejo, pero que también puede implementarse en el formato de la mayoría de los programas de rehabilitación cardíaca mediante el trabajo individual y colectivo de grupo.

Futuras investigaciones utilizando una metodología similar a la de este estudio en la evaluación de las barreras y facilitadores de la prevención cardiovascular a nivel de la atención primaria podrían constituir un nuevo desafío en este importante tema aún no resuelto. 


\section{Referencias}

1. BAMBS CE, SGOMBICH MX, LEIVA LE, BARAONA FE, MARGOZZINI PA, PIZARRO CA, et al. Facilitators and barriers to the adoption of healthy lifestyles after first myocardial infarction in Chile: A qualitative study. Rev Chil Cardiol 2015;34:163-174.

2. FORD ES, AJANI UA, CROFT JB, CRITCHLEY JA, LABARTHE DR, KOTTLE TA, et al. Explaining the Decrease in U.S. Deaths from Coronary Disease, 1980-2000. N Engl J Med 2007;356: 2388-98.

3. GUARDA E, ACEVEDO M, LIRA MT, CHAMORRO G, CORBALÁN R. Prevalence of cardiovascular risk factors among patients suffering vascular events on admission and one year later. Rev Méd Chile 2005;133 :1147-52.

4. CHOW CK, JOLLY S, RAO-MELACINI P, FOX KA, ANAND SS, YUSUF S. Association of diet, exercise, and smoking modification with risk of early cardiovascular events after acute coronary syndromes. Circulation 2010;121:750-8.
5. MURDAUGH CL.The use of grounded theory methodology to study the process of life-style change. Journal of Cardiovascular Nursing 1989, Aspen Publishers, Inc.

6. ROMERO T. Cardiac rehabilitation as a first step in the secondary prevention of coronary heart disease. Rev Méd Chile 2000;128:923-34.

7. ADES PA. Cardiac rehabilitation and secondary prevention of coronary artery disease. N Engl J Med 2001;345: 892- 902

8. Balady GJ, Williams MA, Ades PA, Bittner V, Comoss P, Foody JM, et al. Core components of cardiac rehabilitation/ secondaryprevention programs: 2007 update: a scientific statement from the American Heart Association Exercise, Cardiac Rehabilitation, and Prevention Committee, the Council on Clinical Cardiology; the Councils on Cardiovascular Nursing, Epidemiology and Prevention, and Nutrition, Physical Activity, and Metabolism; and the American Association of Cardiovascular and Pulmonary Rehabilitation. Circulation 2007;115: 2675- 2682. 